\title{
Stimulation of Glycolysis in the Lens by Pyruvate. Implications in Protection against Oxidative Stress
}

\author{
Kavita R Hegde ${ }^{1,3^{*}}$ and Sambhu D Varma ${ }^{1,2}$ \\ ${ }^{1}$ Department of Ophthalmology and Visual Sciences, University of Maryland School of Medicine, Baltimore, USA \\ ${ }^{2}$ Department of Biochemistry and Molecular Biology, University of Maryland School of Medicine, Baltimore, USA \\ ${ }^{3}$ Department of Natural Sciences, Coppin State University, Baltimore, USA
}

"Corresponding author: Kavita Hegde, Associate Professor, Department of Natural Sciences, Coppin State University, West North Avenue, Baltimore, United States, Tel: 410-951-4137; E-mail: khegde@coppin.edu

Rec Date: July 23, 2015; Acc Date: August 14, 2015; Pub Date: August 17, 2015

Copyright: (C) 2015 Hegde and Varma. This is an open-access article distributed under the terms of the Creative Commons Attribution License, which permits unrestricted use, distribution, and reproduction in any medium, provided the original author and source are credited.

\begin{abstract}
Objective: We have previously demonstrated that pyruvate protects the lens against oxidative stress in vitro as well as prevents cataract formation in vivo induced by oxidative stress. The effects have been attributed to its property of scavenging various reactive oxygen species (ROS). Additionally we hypothesize that the preventive effect is also due to its effect of stimulating glycolysis.

Methods: This has been tested as follows: freshly isolated mice lenses were incubated for 4 hours in Tyrode medium containing ${ }^{5}{ }^{3} \mathrm{H}$-glucose as a tracer in the presence and absence of $2 \mathrm{mM}$ sodium pyruvate and determining generation of ${ }^{3} \mathrm{H} 2 \mathrm{O}$ and ${ }^{3} \mathrm{H}$-lactate separated by column chromatography in succession through homemade anion exchange column followed by the phenylboronate and formate mini-columns.

Results: the concentration of ${ }^{3} \mathrm{H}_{2} \mathrm{O}$ in the medium at the end of incubation was $95 \mu \mathrm{M}$ in the controls incubated without pyruvate. In the presence of pyruvate $(2 \mathrm{mM})$ added to the medium, the concentration of ${ }^{3} \mathrm{H}_{2} \mathrm{O}$ attained in the medium was $152 \mu \mathrm{M}$. The corresponding value expressed on the basis of lens weight was 46 nanomoles/lens in the control group vs. 88 nanomoles/lens with $2 \mathrm{mM}$ pyruvate.

Conclusion: As hypothesized, pyruvate was found to stimulate glycolysis in the lens as indicated by the enhanced generation of both ${ }^{3} \mathrm{H}_{2} \mathrm{O}$ and ${ }^{3} \mathrm{H}$-lactate in lenses incubated in its presence as compared to controls. The observed metabolic stimulation is attributed to recycling of NAD generated during the reduction of pyruvate to lactate, NAD being the required cofactor in the oxidation of glyceraldehyde-3-phosphate to 1,3-diphosphoglycerate. That such stimulation is involved in its protective effect is also apparent by our previous reports showing higher ATP levels in the lenses cultured in medium generating ROS in the presence of pyruvate than in its absence, the primary source of ATP in lens being glycolysis.
\end{abstract}

Keywords Glycolysis; Tritiated glucose; Tritiated lactate; Tritiated water; Pyruvate; Oxidative stress; Ion exchange chromatography; Phenylboronate column; NAD/NADH

\section{Introduction}

Generation of reactive oxygen species (ROS) in aqueous humor and lens is well- known to induce oxidative stress to the tissue and consequent cataract formation $[1,2]$. Such species are also implicated in the pathogenesis of many other aging diseases. Although ROS can be generated by several ambient oxidation reactions such as the metal catalyzed auto-oxidation of ascorbate and - $\mathrm{SH}$ containing proteins and glutathione, as well as the leakage of electrons during their transport through the cytochrome chain and its capture by molecular oxygen, the process is significantly augmented in the eye by several photochemically induced pseudocatalytic reactions [3]. Hence, continued penetration of light into the eye, though necessary for vision, has an unwanted side effect of causing aberrant oxidations that lead to many subsequent adverse physiologic and metabolic consequences. Such an effect assumes a greater significance at higher light intensities, especially of the UV frequencies. The visible frequencies can also initiate ROS generation by activation of various redox susceptible components that have absorption maxima in the visible range, such as kynurenine and flavins.

Oxidative stress is known to induce damage not only to lipids and enzymatic and non-enzymatic proteins, but also to DNA. It has been shown that expression of DNA repair genes is altered in lens epithelial cells of age-related cataracts [4]. Cataractous lenses have also been demonstrated to have decrease in the levels of glutathione and thioredoxin, with concomitant decrease in the activities of thioredoxin reductase, thiol transferase and glutathione reductase [5]. Several studies with lens organ culture system as well as with experimental animals have shown that the formation of cataracts can indeed be inhibited by administration of certain oxyradical scavengers such as ascorbate, Vitamin E, and bioflavonoids, to name a few [6-9]. However, the effectiveness of such nutraceuticals can become limited because of their instability due to their spontaneous oxidation, especially by the photochemical reactions initiated in the eye during photopic vision. The products of such oxidation, especially the carbonyls from ascorbate and tocopherols have been suggested to act as potential protein cross linking agents $[10,11]$. They also can become 
pro-oxidant in presence of certain metal ions. Ascorbate has been shown to glycate lens proteins with eventual formation of advanced glycated end products known to be associated with lens aging and cataract formation [12]. Additionally, since ascorbate and tocopherols are not of endogenous nature, they are required to be continuously supplemented in the diet. Epidemiological studies investigating the association of antioxidant vitamins and a lowered incidence of cataracts have yielded results that are yet equivocal [13-15]. Hence we considered the desirability of studying the anti-cataractogenic potential of certain endogenously derived ROS scavengers such as pyruvate, a keto acid metabolite well- known to scavenge various ROS, with rate constants of reaction reaching the diffusion limit $[16,17]$. This is especially true with respect to hydroxyl radical [18]. That pyruvate could be effective against oxidative stress was first suggestible by its effectiveness in prolonging the life of bacteria when exposed to excessive oxygen, the latter being lethal [19]. Such lethality is decreased by pyruvate. It has also been shown to protect the ocular lens against ROS induced damage [20]. Lenses when cultured in media generating ROS by direct addition of hydrogen peroxide, xanthinexanthine oxidase or by photochemical reactions undergo significant physiological damage. This is initially reflected by an inhibition of active cation transport, depletion of GSH, generation of protein carbonyls, lipid peroxidation and loss of ATP [20,21]. Addition of pyruvate to the medium has been shown to be highly protective, the cation transport as well as the biochemistry of the tissue being well maintained. We have hypothesized that the protective effect of pyruvate against ROS induced damage to the lens in vitro and its anticataractogenic effect in vivo is due, in addition to its action as a ROS scavenger, to its action of stimulating tissue metabolism, particularly glycolysis, the main source of ATP in this tissue. The aim of this investigation was hence to further ascertain this possibility. We investigated this hypothesis by culturing mice lenses in presence of ${ }^{5}{ }^{3} \mathrm{H}$-glucose and determining the formation ${ }^{3} \mathrm{H}_{2} \mathrm{O}$ and ${ }^{3} \mathrm{H}$-lactate.

\section{Materials and Methods}

All the chemicals used were obtained from Sigma Chemical Company (St. Louis, MO). Ion exchange (AG1-X8, 200-400 mesh, OH form, Catalog \#143-2446) and phenylboronate (Affi-Gel, Catalog \#153-6103) resins were obtained from Bio-rad laboratories (Hercules, CA). Mice (CD-1, 25-30g.) were obtained from Harlan Laboratories. Animal handling procedures were according to the guidelines prescribed by the Institutional Animal Care and Use Committee and the ARVO statement for the use of animals in research.

Freshly isolated lenses were incubated in $0.5 \mathrm{ml}$ of Tyrode buffer containing $26 \mathrm{mM}$ sodium bicarbonate, $1 \mathrm{mM}$ glucose and trace amounts of $5-{ }^{3} \mathrm{H}$-glucose, without (Controls) and with (Experimentals) the addition of pyruvate $(2 \mathrm{mM})$. The specific activity of $5-{ }^{3} \mathrm{H}$-glucose was $\sim 1400 \mathrm{CPM} /$ nanomole. Incubations were done in a humidified incubator maintained at $37^{\circ} \mathrm{C}$ and gassed with $5 \%$ carbon dioxide in air. At the end of 4 hours, aliquots of the medium were analyzed chromatographically for the contents of ${ }^{3} \mathrm{H}_{2} \mathrm{O}$ as well as ${ }^{3} \mathrm{H}$ lactate generated during incubation, as follows:

Determination of ${ }^{3} \mathrm{H}_{2} \mathrm{O}[22,23]$ this was done by loading $50 \mu \mathrm{l}$ of the medium on a homemade anion exchange column $(4 \times 0.5 \mathrm{~cm}$, AG1X8-OH) piggybacked on a home-made phenylboronate column $(3 \times 0.5 \mathrm{~cm})$. The radioactive glucose and the charged intermediates of glycolysis including the lactate and the glucose present in the medium remained bound to the anion exchange portion of the combined column. Any glucose remaining unbound by the above column gets bound and trapped to the phenylboronate column. The final effluent of the combined column thus contained the ${ }^{3} \mathrm{H}_{2} \mathrm{O}$ generated during the incubation. This was determined by liquid scintillation counting.

Determination of ${ }^{3} \mathrm{H}$-lactate produced: An aliquot of the medium was loaded on a phenylboronate column (which binds glucose) piggybacked on a formate column. The boronate column retains the free glucose including the $5-{ }^{3} \mathrm{H}$-glucose. The formate column retains lactate and other charged components of the glycolysis, including some glucose leaked out of the boronate column. The columns were then eluted with $\mathrm{dH}_{2} \mathrm{O}$ to get rid of the ${ }^{3} \mathrm{H}_{2} \mathrm{O}$ as well as any free glucose that might have been unbound on the column. The boronate column was then detached, and lactate including ${ }^{3} \mathrm{H}$-lactate generated during incubation was recovered by elution with $0.1 \mathrm{M}$ formic acid and quantified by liquid scintillation counting.

\section{Results}

Several previous investigations on lens metabolism have assessed the status of glycolysis by incubating the tissue in various media and determining change in medium lactate $[24,25]$. A decrease in medium lactate with time of incubation, relative to a control is taken as an index of the inhibition of glycolysis. Since glycolysis includes several reactions representing the Embden-Myerhoff pathway, such measurement of lactate provides only limited information on the status of overall process of glycolysis, unless lactate determination is combined with the determinations of several other substrates generated over the specific period of experiment. Quantitation of lactate actually produced during incubation also needs to be corrected for the endogenous lactate that pre-exists in the lens prior to incubation [26]. In the present investigations, it was hypothesized that the beneficial effect of pyruvate in protecting the lens against oxidative stress could also be due to metabolic stimulation caused by recycling of NAD generated during the reduction of pyruvate to lactate, NAD being the required co-substrate in the oxidation of glyceraldehyde-3phosphate to 1,3-diphosphoglycerate. The reaction is also accompanied with ATP generation by substrate phosphorylation and simultaneous generation of 3-phosphoglycerate. The intra-molecular dismutation of the latter produces 2-phosphoglycerate, which generates water and phosphoenol pyruvic acid, another major highenergy compound generating ATP as well as pyruvate. The stimulation of glucose metabolism via glycolysis caused by exogenously added pyruvate in the medium was hence hypothesized to be associated with generation of water. This was conceived to be reflected by an increase in the amount of ${ }^{3} \mathrm{H}_{2} \mathrm{O}$ starting with $5-{ }^{3} \mathrm{H}$ glucose. That has been found to be true.

As shown in Figure 1, the concentration of ${ }^{3} \mathrm{H}_{2} \mathrm{O}$ in the medium at the end of incubation was $95 \mu \mathrm{M}$ in the controls incubated without pyruvate. The corresponding value expressed on the basis of lens weight was 46 nanomoles/ lens Figure 2 . In the presence of pyruvate (2 $\mathrm{mM}$ ) added to the medium, the concentration of ${ }^{3} \mathrm{H}_{2} \mathrm{O}$ attained in the medium was $152 \mu \mathrm{M}$. Expressed on the basis of lens weight, ${ }^{3} \mathrm{H}_{2} \mathrm{O}$ produced was 88 nanomoles/lens. Hence, formation of ${ }^{3} \mathrm{H}_{2} \mathrm{O}$ is substantially higher when the lenses were incubated with pyruvate, expressed as its concentration in the medium as well as when calculated on the basis of lens weight. The results therefore provide convincing evidence of the possibility that pyruvate offers protection to the lens under stress situations by accelerating glycolysis, in addition to its oxyradical scavenging function. 
Citation: Hegde KR, Varma SD (2015) Stimulation of Glycolysis in the Lens by Pyruvate. Implications in Protection against Oxidative Stress. J

This possibility was examined further by measuring the formation of ${ }^{3} \mathrm{H}$-lactate. As shown in Figure 3 concentration of the ${ }^{3} \mathrm{H}$-lactate derived from $5-{ }^{3} \mathrm{H}$ glucose was $8.6 \mu \mathrm{M}$. The amount expressed on the per lens basis was 4.3nanomoles/lens Figure 4. In experiments where incubations were done with pyruvate, the medium lactate concentration rose to $\sim 13 \mu \mathrm{M}$. The value on the per lens basis was 6.5 nanomoles. Hence it was about 1.5 times more than that in the absence of pyruvate. These results hence further prove the stimulation of glycolysis by exogenous enrichment with this compound.

Fig. 1

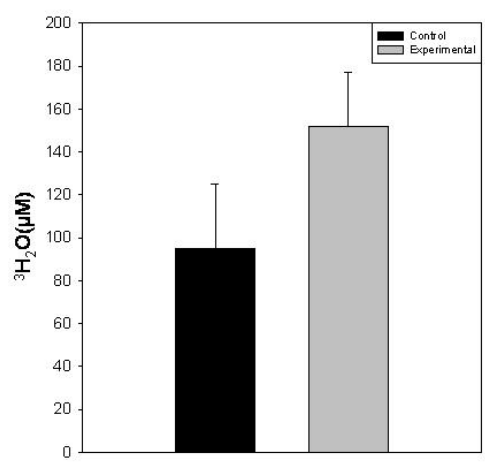

Figure 1: Concentration of ${ }^{3} \mathrm{H}_{2} \mathrm{O}$ generated in the medium of incubation. Mice (CD-1) lenses were incubated in Tyrode medium containing $5-3 \mathrm{H}$ glucose for 4 hours. The medium was then analyzed for the content of ${ }^{3} \mathrm{H}_{2} \mathrm{O}$ as described in the text. The results are expressed on the basis of $\mu \mathrm{M}$ concentration in the medium, the values representing the mean \pm S.D. of at least 6 lenses. The amount of ${ }^{3} \mathrm{H}_{2} \mathrm{O}$ generated in the presence of pyruvate is significantly higher as compared to that in its absence, $\mathrm{p}<0.001$.

\section{Fig. 2}

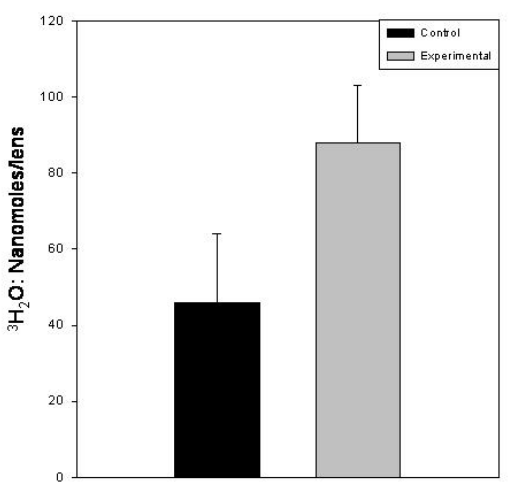

Figure 2: ${ }^{3} \mathrm{H}_{2} \mathrm{O}$ generation as expressed on the basis of nanomoles per lens. Lenses were incubated as described in the Materials and Methods section. The values represent the mean \pm S.D. of $\geq 6$ lenses; $\mathrm{p}<0.001$ between the control and experimental groups.

\section{Fig. 3}

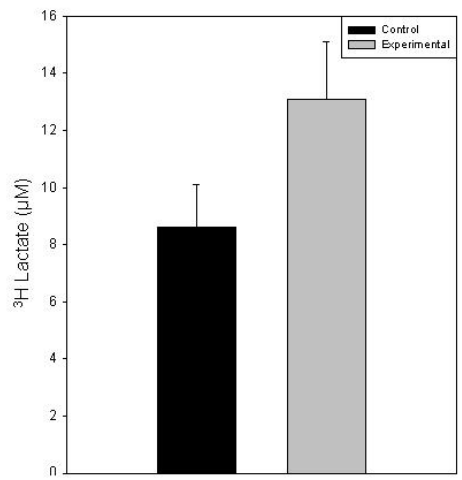

Figure 3: Concentration of ${ }^{3} \mathrm{H}$-lactate in the incubation medium: CD-1 mice lenses were incubated in Tyrode medium containing $5-{ }^{3} \mathrm{H}$-glucose in the absence (Control) and presence (Experimental) of $2 \mathrm{mM}$ pyruvate for 4 hours. The post-incubation medium was analyzed for the content of lactate following its elution from a formate column and liquid scintillation counting, as described in the text. The results are expressed as the mean \pm S.D. of at least 6 lenses; $\mathrm{p}<0.001$ between control and experimental. 


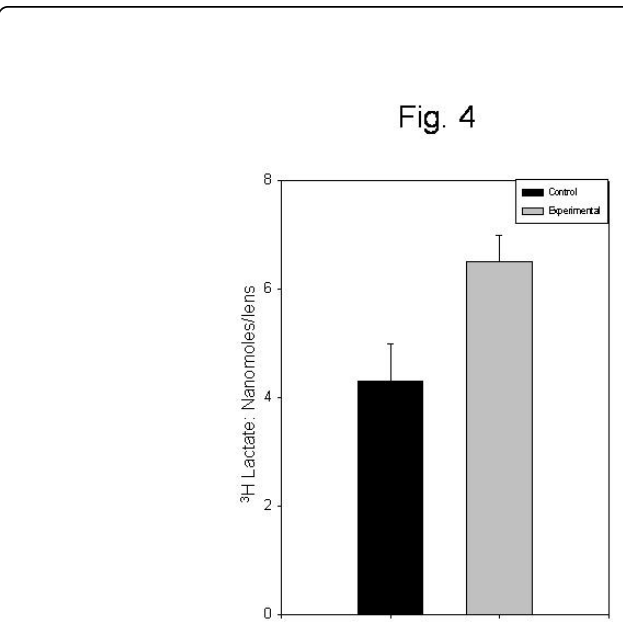

Figure 4: Generation of ${ }^{3} \mathrm{H}$-lactate expressed as nanomoles per lens: The values represent the mean \pm S.D. of $\geq 6$ lenses; $p<0.001$.

\section{Discussion}

Previous studies have shown that the bioenergetic support to the lens is primarily provided by glycolysis. A number of such studies have shown that this pathway becomes sluggish with aging as well as with certain diseases including diabetes and galactosemia. This is reflected by decreases in the contents of lens lactate as well as ATP. In addition, decrease in the activity of glyceraldehyde-3-phosphate dehydrogenase has also been shown to occur in aging and cataractous lenses [5]. Comparison of metabolomic profiles of normal and cataractous lenses using high-frequency nuclear magnetic resonance (NMR) and highperformance liquid chromatography with high-resolution massspectrometric detection (LC-MS) methods has revealed a lowered metabolic activity in cataractous lenses, as suggested by decrease in the levels of almost all metabolites in the cataractous lens vs. normal lens [27].

Inactivation of glyceraldehyde-3-phosphate dehydrogenase, a key glycolytic enzyme, as well as others is highly likely to cause inhibition of tissue glycolysis and other metabolic pathways. Such inactivation can be induced by oxidation of several enzymes by oxyradicals generated during photochemical oxidation of certain lens constituents, as well as during many metal ion dependent auto-oxidative reactions that proceed ambiently. Hence inhibition of cataract formation would require the presence of oxyradical scavengers as a first line of defense. A second line of defense would be to use compounds that have the ability to overcome the accompanying metabolic inhibitions, by acting as metabolic substrates. Previous studies have demonstrated that pyruvate inhibits the cataractogenic process in diabetic animals [28-30]. It is quite likely that it provides the lens a first line of defense via its oxyradical scavenging properties and a second line of defense by stimulating glycolysis. It is conceived that it can potentially do the latter by maintaining a consistent supply of NAD generated by various $\mathrm{NADH}$ dependent dehydrogenation reactions, including $\mathrm{NADH}$ dependent reduction of the exogenously supplied pyruvate. That its conversion to lactate is a favorable reaction is also apparent from its $\Delta \mathrm{G}$ values of $-6 \mathrm{kcal} / \mathrm{mole}$ [31]. Hence the possibility that increasing the amount of pyruvate would enhance NAD generation is feasible thermodynamically also. Additional generation of NAD is expected to stimulate glycolysis by driving the glyceraldehyde-3-phosphate dehydrogenase reaction in the direction of oxidation, converting glyceraldehyde-3-phosphate to 1,3-diphosphoglycerate with eventual stimulation of ATP formation. The possibility that pyruvate can indeed accomplish such enhancement of glycolysis is convincingly demonstrable by incubation of the lenses with $5-{ }^{3} \mathrm{H}$ glucose and determining the formation of titrated water chromatographically as described above. Passage of the incubation medium through anion exchange column in tandem with the boronate column removes most of the expected glycolytic metabolites and unused glucose, ensuring that the radioactivity in the final eluate is due to the water simultaneously generated in the enolase-catalyzed reaction that converts 2-phosphoglycerate to phosphoenolpyruvate. Hence measurement of the amount of ${ }^{3} \mathrm{H}_{2} \mathrm{O}$ and its increase or decrease under various conditions provides a strong means of detecting any alteration in the glycolytic activity of a tissue under examination. The increase in the formation of ${ }^{3} \mathrm{H}_{2} \mathrm{O}$ by the lenses incubated with ${ }^{5-}{ }^{3} \mathrm{H}$ glucose in the presence of pyruvate, especially associated also with the increase in the contents of ${ }^{3} \mathrm{H}$-lactate, therefore provides a more convincing evidence of enhancement in the glycolytic activity of the lens than the measurement of lactate alone from pyruvate, which can be reduced to lactate by NADH and NADPH generated from several glycolytic as well as non-glycolytic reactions. Mechanistically, formation of water during the conversion of 2-phoshoglycerate (2PGA) to phosphoenolpyruvate requires the formation of an initial $\mathrm{Mg}^{2+}$ enolase complex. Combination of this complex with 2-PGA then causes dissociation of an acidic proton from C-2. This combines with the nucleophilic $-\mathrm{OH}$ base of $-\mathrm{CH}_{2} \mathrm{OH}$ at $\mathrm{C}-3$ of 2 -PGA, to be followed by the splitting of a water molecule with simultaneous generation of phosphoenolpyruvate. The free energy change of this reaction is reasonably small $(\sim 1 \mathrm{kcal})$, so that the reaction can proceed at physiological temperature without any thermodynamic hindrance. The results obtained showing an increase in the ${ }^{3} \mathrm{H}_{2} \mathrm{O}$ from $5-{ }^{3} \mathrm{H}$ glucose in presence of pyruvate is also strongly supported by the isotopic exchange studies [32], as well as by crystal structure of the enzyme- $\mathrm{Mg}^{2+}$ complex [33]. $\mathrm{Mg}^{2+}$ is a well-known essential cofactor required for the activity of enolase.

The results therefore strongly suggest that the protective effect of pyruvate against cataract formation induced by ROS generation as well as by diabetic conditions is attributable to its action as a metabolic agonist, supporting glycolysis. This is in addition to its effectiveness in scavenging ROS. Unlike many other scavengers of ROS, the products of the reaction of pyruvate with ROS are not toxic. The possibility that it may have other beneficial effects such as competitive inhibition of polyol synthesis and protein glycation has also been shown before.

\section{Acknowledgements}

The authors are thankful to NEI, NIH, and Research to Prevent Blindness Inc., for their financial support, and to Svitlana Kovtun for technical assistance.

\section{References}

1. Varma SD, Ets TK, Richards RD (1977) Protection against superoxide radicals in rat lens. Ophthalmic Res 9: 421-431.

2. Zigler JS Jr, Goosey JD (1984) Singlet oxygen as a possible factor in human senile nuclear cataract. Curr Eye Res 3: 39-45.

3. Varma SD, Kumar S, Richards RD (1979) Light induced damage to ocular lens cation pump. Prevention by Vitamin C. Proc Natl Acad Sci 76: 3504-3506. 
Citation: Hegde KR, Varma SD (2015) Stimulation of Glycolysis in the Lens by Pyruvate. Implications in Protection against Oxidative Stress. J Metabolic Synd 4: 179. doi:10.4172/2167-0943.1000179

Page 5 of 5

4. Li F, Wang Y, Zhang G, Zhou J, Yang L, et al. (2014) Expression and methylation of DNA repair genes in lens epithelium cells of age-related cataract. Mutat Res. 766-767: 31-36.

5. Wei M, Xing KY, Fan YC, Libondi T, Lou MF (2014) Loss of thiol repair systems in human cataractous lenses. Invest Ophthalmol Vis Sci 56: 598-605.

6. Hegde KR, Varma SD (2004) Protective effect of ascorbate against oxidative stress in the mouse lens. Biochim Biophys Acta 1670: 12-18.

7. Trevithick JR, Linklater HA, Mitton KP, Dzialoszynski T, Sanford SE (1989) Modeling cortical cataractogenesis: IX. Activity of vitamin E and esters in preventing cataracts and gamma-crystallin leakage from lenses in diabetic rats. Ann N Y Acad Sci 570: 358-371.

8. Ayala MN, Söderberg PG (2004) Vitamin E can protect against ultraviolet radiation-induced cataract in albino rats. Ophthalmic Res 36: 264-269.

9. Varma SD, Mizuno A, Kinoshita JH (1977) Diabetic cataracts and flavonoids. Science 195: 205-206.

10. Nagaraj RH, Monnier VM (1992) Isolation and characterization of a blue fluorophore from human eye lens crystallins: in vitro formation from Maillard reaction with ascorbate and ribose. Biochim Biophys Acta 1116: $34-42$.

11. Cheng R, Lin B, Lee KW, Ortwerth BJ (2001) Similarity of the yellow chromophores isolated from human cataracts with those from ascorbic acid-modified calf lens proteins: evidence for ascorbic acid glycation during cataract formation. Biochim Biophys Acta 1537: 14-26.

12. Smuda M, Henning C, Raghavan CT, Johar K, Vasavada AR, et al. (2015) Comprehensive analysis of maillard protein modifications in human lenses: effect of age and cataract. Biochemistry 54: 2500-2507.

13. Leske MC, Chylack LT Jr, He Q, Wu SY, Schoenfeld E, et al. (1998) Antioxidant vitamins and nuclear opacities: the longitudinal study of cataract. Ophthalmology 105: 831-836.

14. Jacques PF, Hartz SC, Chylack LT Jr, McGandy RB, Sadowski JA (1988) Nutritional status in persons with and without senile cataracts: blood vitamin and mineral levels. Am J Clin Nutr 48: 152-158.

15. Taylor A, Jacques PF, Chylack LT Jr, Hankinson SE, Khu PM, et al. (2002) Long-term intake of vitamins and carotenoids and odds of early age-related cortical and posterior subcapsular lens opacities. Am J Clin Nutr 75: 540-549.

16. Fenton HJH, Jones HO (1900) Oxidation of organic acids in presence of ferrous iron, Part I. J Chem Soc Trans 77: 69-76.

17. Holleman AF (1904) Note on the action of oxygenated water on alphaketo acids and 1,2 dicetones. Recl Tran Chi Pays Bas Belq 23: 169-172.
18. Ervens B, Gligorovski S, Herrmann H (2003) Temperature dependent rate constants for hydroxyl radical reactions with organic compounds in aqueous solutions. Phys Chem Chem Phys 5: 1811-1824.

19. Sevag MG, Maiweg L (1934) The respiration mechanism of pneumococcus III. J Exp Med 60: 95-105.

20. Varma SD, Morris SM (1988) Peroxide damage to the eye lens in vitro prevention by pyruvate. Free Rad Res Comm 4: 283-290.

21. Varma SD, Hegde K, Henein M (2003) Oxidative damage to mouse lens in culture. Protective effect of pyruvate. Biochim Biophys Acta 1621: 246-252.

22. Clark DG, Rongstad R and Katz J (1973). Isotopic evidence of futile cycles in liver cells. Biochem Biophys Res Comm 54: 1141-1148.

23. Allard MF, Schönekess BO, Henning SL, English DR, Lopaschuk GD (1994) Contribution of oxidative metabolism and glycolysis to ATP production in the hypertrophied heart. Am J Physiol Heart Circ Physiol 267: H742-H750.

24. Van Heyningen R (1965) The metabolism of glucose by rabbit lens in the presence and absence of oxygen. Biochem J 96: 419-431.

25. Hightower KR and Harrison SE (1987) The influence of calcium on glucose metabolism in the rabbit lens. Invest Ophthalmol Vis Sci 28: 1433-1436.

26. Gillis KM, Chylack LT Jr, Cheng HM (1981) Age and the control of glycolysis in the rat lens. Invest Ophthalmol Vis Sci 20: 457-466.

27. Tsentalovich YP, Verkhovod TD, Yanshole VV, Kiryutin AS, Yanshole $\mathrm{LV}$, et al. (2015) Metabolomic composition of normal aged and cataractous human lenses. Exp Eye Res 134: 15-23.

28. Varma SD, Ramachandran S, Devamanoharan PS, Morris SM, Ali AH (1995) Prevention of oxidative damage to rat lens by pyruvate in vitro: possible attenuation in vivo. Curr Eye Res 14: 643-649.

29. Zhao W, Devamanoharan PS, Henein M, Ali AH, Varma SD (2000) Diabetes-induced biochemical changes in rat lens: attenuation of cataractogenesis by pyruvate. Diabetes Obes Metab 2: 165-174.

30. Hegde KR, Varma SD (2005) Prevention of cataract by pyruvate in experimentally diabetic mice. Mol Cell Biochem 269: 115-120.

31. West ES, Todd WR, Mason HS, Van Bruggen JT (1970) Textbook of Biochemistry. The Macmillan Company, London.

32. Dinovo EC, Boyer PD (1971) Isotopic Probes of the Enolase Reaction Mechanism. J Biol Chem 246: 4586-4593.

33. Lebioda L, Stec B (1991) Mechanism of enolase: the crystal structure of enolase-Mg2(+)2phosphoglycerate/phosphoenolpyruvate complex at 2.2A resolution 30: 2817-2822. 